\title{
A batch-by-batch free route for the continuous production of black phosphorus nanosheets for targeted combination cancer therapy
}

\author{
Bijay Kumar Poudel', Jungho Hwang ${ }^{1}$, Sae Kwang Ku${ }^{2}$, Jong Oh Kim ${ }^{3}$ and Jeong Hoon Byeon $\mathbb{D}^{4}$
}

\begin{abstract}
Although 2D materials such as graphenes, chalcogenides, and black phosphorus (BP) have been intensively studied for a wide range of future technological applications, the multiple harsh reactions and post-treatments required to produce exfoliated nanosheets (NSs) represent challenging barriers to their realization. In the present study, a batchby-batch free route to produce BP NSs was demonstrated, and the NSs were employed as base materials for the chemo-phototherapy of breast cancer. Specifically, a single-pass catalytic conversion of an all-in-one precursor (red $\mathrm{P}$, $\mathrm{Au}-\mathrm{Sn}$, and iodine) in a heated tubular reactor (at a $650^{\circ} \mathrm{C}$ wall temperature for $15.5 \mathrm{~s}$ ) continuously produced crumpled BP NSs, and the NS-laden gas stream was passed through an activated carbon-packed tube to remove the reacted gases. Subsequently, doxorubicin (D), poly-L-lysine $(L)$, and hyaluronic acid $(H)$ were incorporated without further purification of these NSs to form BP-DLH, which was then employed in both in vitro and in vivo chemophototherapies. The biocompatibility and near-infrared-induced photothermal activity of BP NSs facilitate targeted delivery to CD44-overexpressing breast cancer cells to assist in the therapeutic efficacy of D.
\end{abstract}

\section{Introduction}

In recent years, the explosive rise of multidisciplinary interest in graphene has also stimulated the study of other two-dimensional (2D) planar materials with diverse functionalities, such as transition metal dichalcogenides (TMDs; e.g., $\mathrm{MoS}_{2}, \mathrm{WS}_{2}, \mathrm{MoSe}_{2}$, and $\mathrm{MoTe}_{2}$ ), 2D-Xenes (silicene, germanene, and stanine), boron nitride, $\mathrm{Bi}_{2} \mathrm{Te}_{3}$, $\mathrm{GaSe}$, and $\mathrm{MnO}_{2}{ }^{1,2}$. Since the isolation of thin layers of black phosphorus (BP) via micromechanical cleavage was first reported ${ }^{3,4}$, interest in BP, which was discovered a century ago, has reignited. Phosphorene, the monolayer counterpart of $\mathrm{BP}$, is an atomically thin, $2 \mathrm{D}, p$-type,

Correspondence: Jungho Hwang (hwangjh@yonsei.ac.kr) or Jong

Oh Kim (jongohkim@yu.ac.kr) or Jeong Hoon Byeon (postjb@yu.ac.kr)

${ }^{1}$ School of Mechanical Engineering, Yonsei University, Seoul 03722, Republic of Korea

${ }^{2}$ College of Korean Medicine, Daegu Haany University, Gyeongsan 38610, Republic of Korea

Full list of author information is available at the end of the article. flexible semiconductor material with a puckered honeycomb lattice structure. It possesses an intrinsic direct bandgap energy that shifts from $0.3 \mathrm{eV}$ (bulk BP) to $2.0 \mathrm{eV}$ (monolayers) as a function of the number of layers. This can be useful for infrared optoelectronics to bridge the gap between bandgap-less graphene and large-bandgap TMDs $(>1.2 \mathrm{eV})^{3}$. Unlike TMDs, phosphorene exhibits superior carrier mobility, with an anisotropic hole mobility of up to $1000 \mathrm{~cm}^{2} \mathrm{~V}^{-1} \mathrm{~s}^{-1}$ and on/off current ratios of $10^{4}-10^{5}$ at room temperature ${ }^{3,5}$. These properties, along with its good electrical conductivity $\left(300 \mathrm{~S} \mathrm{~m}^{-1}\right)$, excellent mechanical properties, large interlayer size, fast ion diffusion, photothermal activity, and thermodynamic stability, make BP a very promising candidate for applications in nanoelectronics, photovoltaics, spintronics, biosensing, and energy storage ${ }^{6}$.

More recently, several reports have highlighted the utility of BP in biomedical applications. PEGylated BP

\section{(c) The Author(s) 2018}

(c) Open Access This article is licensed under a Creative Commons Attribution 4.0 International License, which permits use, sharing, adaptation, distribution and reproduction in any medium or format, as long as you give appropriate credit to the original author(s) and the source, provide a link to the Creative Commons license, and indicate if changes were made. The images or other third party material in this article are included in the article's Creative Commons license, unless indicated otherwise in a credit line to the material. If material is not included in the article's Creative Commons license and your intended use is not permitted by statutory regulation or exceeds the permitted use, you will need to obtain permission directly from the copyright holder. To view a copy of this license, visit http://creativecommons.org/licenses/by/4.0/. 


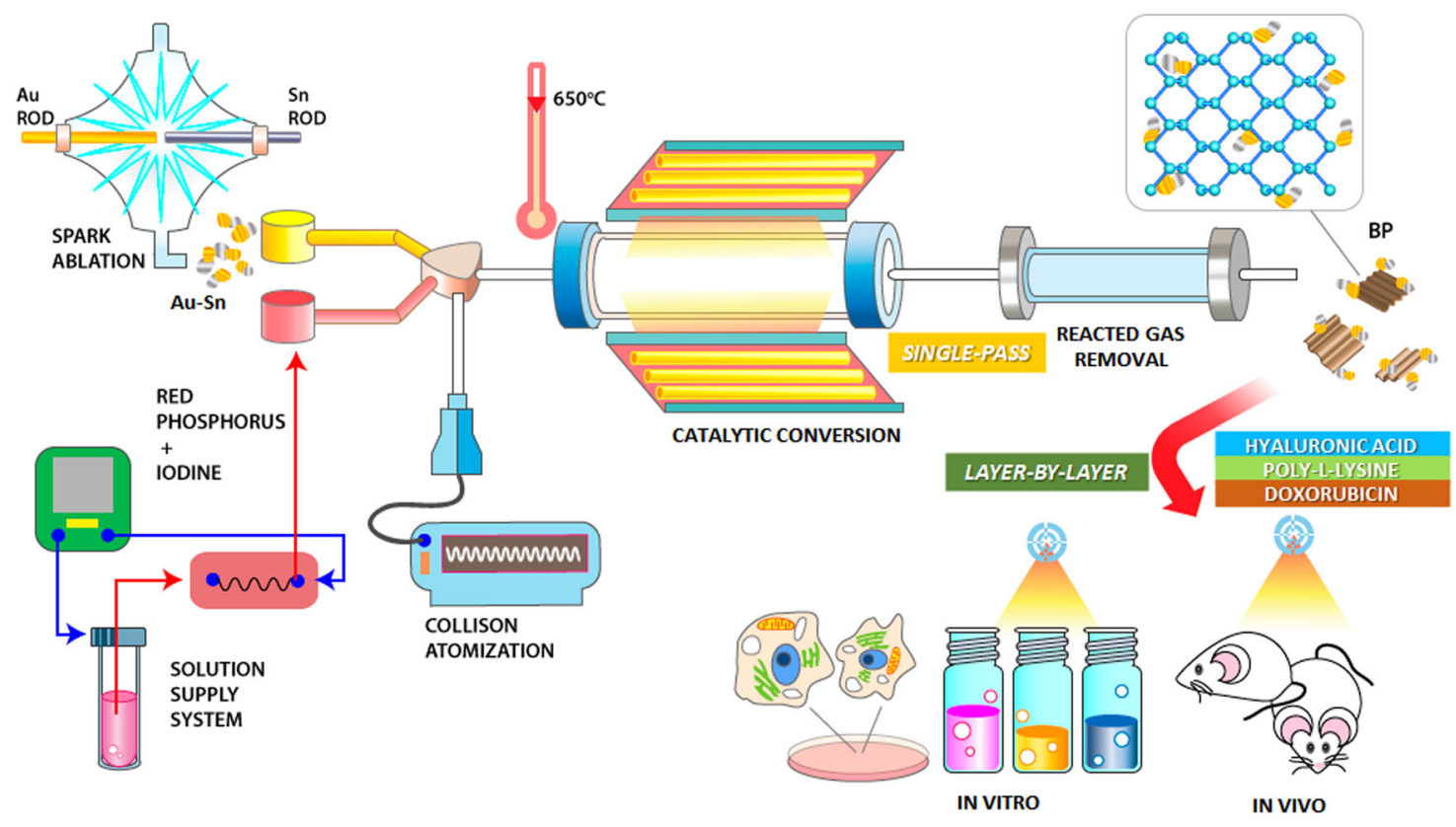

Fig. 1 Conceptual diagram of single-pass BP synthesis and subsequent DLH loading. Spark-produced Au-Sn nanoparticle-laden nitrogen gas flow was employed as the operating gas for collison atomization of RP-I solution, where gas pressurization near the orifice induced insertion of $\mathrm{Au}-\mathrm{Sn}$ into the RP-I solution to produce the all-in-one precursor (RP-I+Au-Sn) droplets. The droplets were then injected into a heated tubular reactor $\left(650^{\circ} \mathrm{C}\right.$ wall temperature) for the catalytic conversion of $\mathrm{RP}$ into $\mathrm{BP}$, and the reacted gases were removed by flowing the mixture through an activated carbon-packed hollow tube. DLH was incorporated into the collected BP to form BP-DLH via an LbL process, and the BP-DLH was then employed in targeted chemo-phototherapy experiments to determine its therapeutic efficacy

exhibits broad absorption spectra across the UV and nearinfrared (NIR) regions, and exhibits a higher photothermal conversion efficiency than gold nanorods, making it a promising candidate for photothermal therapy and biosensing ${ }^{7,8}$. BP has also been demonstrated to be an efficient photosensitizer for the photodynamic therapy of cancer because it can generate singlet oxygen with a high quantum yield upon NIR irradiation ${ }^{9}$. The large surface area of BP nanosheets (NSs) makes them a feasible delivery platform to incorporate drug/imaging molecules, as both sides are accessible for loading ${ }^{10}$. BP NSs have also been reported to be highly biocompatible, biodegradable, and photostable ${ }^{11}$. However, despite the substantial potential of BP for future theranostic applications, the study of BP in cancer therapy and diagnostics is still in its infancy.

One reason for this may be the difficulty of preparing BP NSs. For instance, the chemical conversion of red phosphorus (RP) into BP requires multiple complex and time-consuming steps, and harsh chemicals and reaction conditions (high pressure and temperature). The process also requires purification steps to remove unwanted byproducts and unconsumed reactants ${ }^{12,13}$. Post-treatments, such as mechanical shearing and shear/ultrasonic exfoliation, can produce thin-layered BP (i.e., BP NSs) from bulk BP; however, these approaches produce low yields
$(<45 \%)$ and throughput, resulting in amounts that might only be sufficient for research purposes ${ }^{14}$. Moreover, chemical exfoliation requires the extensive use of toxic and costly solvents, such as $\mathrm{N}$-methyl pyrrolidone, $\mathrm{N}, \mathrm{N}$ dimethylformamide or benzonitrile, which necessitate additional treatments before the BP NSs can be used for bioapplications ${ }^{14-17}$. Utilizing BP for practical applications, therefore, requires the development of a practical preparation strategy.

In the present study, a batch-by-batch free route to continuously producing BP NSs via single-pass catalytic conversion was developed, and the resulting NSs were directly employed without exfoliation, purification or post-treatment to incorporate active pharmaceutical ingredients. The ingredients used were doxorubicin (D, model drug), poly-L-lysine (L, cationic component), and hyaluronic acid $(\mathrm{H}$, a targeting agent for CD44overexpressing cells, which is a common property of highly malignant and chemo-resistant tumors ${ }^{18-20}$ ). A diagram of this process is shown in Fig. 1. Briefly, an allin-one precursor $(\mathrm{RP}-\mathrm{I}+\mathrm{Au}-\mathrm{Sn})$ containing $\mathrm{RP}$, iodine $(\mathrm{I})$, and gold $(\mathrm{Au})$-tin $(\mathrm{Sn})$ was prepared under a gas stream via a serial combination of spark ablation (between $\mathrm{Au}$ and Sn rods) and collison atomization (using RP-I solution). Au-Sn in the flowing precursor acted as a catalyst for BP formation based on principles described 
previously ${ }^{12,21}$. The precursor entered a heated tubular reactor operating with a wall temperature of $650{ }^{\circ} \mathrm{C}$ for the in-flight catalytic conversion (15.5 s reaction time) of $\mathrm{RP}$ into BP. BP was then used as a base material to load D, $\mathrm{L}$, and $\mathrm{H}$ through an aqueous layer-by-layer (LbL) process (Figure S1) to form BP-DLH. This material was tested for efficacy in targeted chemo-phototherapy both in vitro (using both MCF-7 and MDA-MB-231 breast cancer cell lines) and in vivo (using MDA-MB-231 cell-implanted $\mathrm{BALB} / \mathrm{c}$ mice). To evaluate the therapeutic potential of $\mathrm{BP}$, its biocompatibility, $\mathrm{pH}$ - and NIR-triggered drug release activity, CD44 receptor targetability, and accumulation in cancer cells were assessed.

\section{Materials and methods \\ BP and BP-DLH preparation}

BP NSs were prepared through the catalytic conversion of RP in an all-in-one precursor (RP, $\mathrm{Au}-\mathrm{Sn}$, and iodine) droplet at a wall temperature of $650{ }^{\circ} \mathrm{C}$ under a gas stream (Fig. 1). Au-Sn nanoparticles in the precursor droplets were selected as catalysts for the conversion, where an ambient spark ablation between Au (anode; AU-172561, Nilaco, Japan)-Sn (cathode; SN-441565, Nilaco, Japan) rods $\left(30 \mathrm{kV} \mathrm{cm}^{-1}\right)$ was employed under nitrogen gas flow $\left(1.57 \mathrm{~L} \mathrm{~min}^{-1}\right)$. The $\mathrm{Au}-\mathrm{Sn}$-laden nitrogen gas flow was directly injected into a collison atomizer containing RP-I [100 mg RP (Alfa Aesar, USA) and $3.5 \mathrm{mg}$ I (Sigma Aldrich, US) in $50 \mathrm{~mL}$ ethanol] solution, which was used as the operating gas for spraying the solution. During this process, $\mathrm{Au}-\mathrm{Sn}$ nanoparticles were inserted into the RP-I solution via gas pressurization near the orifice of the atomizer, resulting in the formation of a hybrid droplet that acted as an all-in-one precursor. The precursor flow was then injected into a heated tubular reactor $\left(650^{\circ} \mathrm{C}\right.$, $15.5 \mathrm{~s}$ residence time) for the catalytic conversion of RP into BP, followed by injection into an activated carbonpacked hollow tube to remove reacted gases from the flow. Lastly, BP in the flow was separated via electrostatic charging-collection and kept in its dried form for loading DLH via the LbL process (Figure S1) without further purification.

First, $3 \mathrm{~mL}$ of BP dispersion $\left(1 \mathrm{mg} \mathrm{mL}^{-1}\right)$ was prepared, which was then mixed with $3 \mathrm{~mL}$ of $\mathrm{D}$ (Dong-A Pharmaceutical Company, South Korea) solution $\left(4 \mathrm{mg} \mathrm{mL}^{-1}\right)$ and stirred overnight at room temperature. The solution was centrifuged at $5000 \mathrm{rpm}$, excess D was decanted off, and the volume was set to $1 \mathrm{~mL}$ by adding deionized water. Then, $0.2 \mathrm{~mL}$ of $\mathrm{L}\left(1600 D_{\mathrm{a}}\right.$; Alamanda Polymers Co., USA) solution $\left(5 \mathrm{mg} \mathrm{mL}^{-1}\right)$ was added, and the mixture solution was vortexed and sonicated in a bath sonicator for $0.5 \mathrm{~h}$. Excess $\mathrm{L}$ was removed by centrifugation and washed with deionized water. The volume of solution was set again to $1 \mathrm{~mL}$. Then, $0.5 \mathrm{~mL}$ of $\mathrm{H}(3000$ $D_{\mathrm{a}}$; B\&K Technology, China) solution $\left(5 \mathrm{mg} \mathrm{mL}^{-1}\right)$ was added, and bath sonication was applied for $0.5 \mathrm{~h}$. Excess $\mathrm{H}$ was removed by centrifugation and washed to form BPDLH. The sample was stored at $4{ }^{\circ} \mathrm{C}$ for further investigation.

\section{Characterization \\ Size distribution}

The mobility equivalent diameters of $\mathrm{BP}$ and precursor $(\mathrm{RP}-\mathrm{I}+\mathrm{Au}-\mathrm{Sn})$ in the gas stream were analyzed using a scanning mobility particle sizer (SMPS, 3936, TSI, USA), which consisted of a differential mobility analyzer (3081, TSI, USA), a condensation particle counter (3776, TSI, USA), and an aerosol charge neutralizer (4530, HCT, Korea). It was operated at flow rates of 0.1 and $1.0 \mathrm{~L}$ $\mathrm{min}^{-1}$ for sampling and sheathing, respectively, and the scan time was $135 \mathrm{~s}$. The hydrodynamic diameters of BP and precursor dispersed in phosphate buffered saline (PBS) were measured using dynamic light scattering (DLS, Nano-ZS, Malvern Instruments, UK) with a He-Ne laser $(\lambda=663 \mathrm{~nm})$ and a scattering angle of $90^{\circ}$ at $25^{\circ} \mathrm{C}$.

\section{Morphology}

Morphological analyses were performed on samples (BP and BP-DLH) directly collected on 200-mesh carboncoated copper grids (Tedpella, USA) using TEM grid filtration (Ineris, France). The grids were observed by transmission electron microscopy (TEM, Tecnai $G^{2}$ F20 S-TWIN, FEI, USA) to confirm the successful preparation of BP and BP-DLH. Three-dimensional imaging of the samples deposited on a mica plate was performed using atomic force microscopy (AFM, Nanoscope IIIa, Digital Instruments Co., USA) in tapping mode with a commercial pyramidal silicon tip with a radius of $10 \mathrm{~nm}$ and a nominal force constant of $0.1 \mathrm{~N} \mathrm{~m}^{-1}$.

\section{Thermogravimetric, surface, and optical properties}

To measure the contents of overlayered $\mathrm{L}$ and $\mathrm{H}$, thermogravimetric analysis (TGA) was performed using a PerkinElmer Diamond TG/DTA instrument under nitrogen from room temperature to $900^{\circ} \mathrm{C}\left(10^{\circ} \mathrm{C} \mathrm{min}{ }^{-1}\right)$. To examine the surface chemistry of the samples, the prepared samples were directly deposited on polytetrafluoroethylene substrates (11807-47-N, Sartorius, Germany) for analysis by Fourier transform infrared spectroscopy (FTIR, iS-10, Thermo Electron, USA) in absorbance mode (range: $600-4000 \mathrm{~cm}^{-1}$ ). The surface structures of the samples deposited on glass disks were analyzed using Raman spectroscopy (XploRA Plus, HORIBA, Japan) and X-ray photoelectron spectroscopy (XPS; Axis-HIS, Kratos Analytical, Japan). Light absorption spectra of the samples dispersed in PBS were measured using UV-Vis spectroscopy (T60, PG Instruments, $\mathrm{UK})$. 


\section{Photothermal activity}

The NIR irradiation-induced temperature elevation of the BP dispersion was analyzed using an $808 \mathrm{~nm}$ fibercoupled IR diode laser module (FC-W-808 nm-30 W, Changchun New Industries Optoelectronics Technology, China) in continuous wave operating mode at a fixed distance of $10 \mathrm{~cm}$. Temperature contours and thermal images were recorded using a thermal camera (ThermApp TH, Opgal Optronic Industries Ltd., Israel).

\section{In vitro $D$ release}

The in vitro release profile of D from BP-DLH was determined at pH 5.5 [acetate buffered saline (ABS), 0.14 $\mathrm{M} \mathrm{NaCl}, 0.1 \%$ Tween 20] and pH 7.4 (PBS, 0.14 M NaCl, $0.1 \%$ Tween 20) using dialysis membrane tubing. The chosen concentrations of BP-DLH were placed in a dialysis bag (MWCO: 4000-6000 $D_{\mathrm{a}}$, Spectra/Por ${ }^{\circledR}$, USA), sealed at both ends and immersed in a tube $(50 \mathrm{~mL})$ containing $20 \mathrm{~mL}$ of ABS or PBS. The construct was placed in a water bath shaker (HST-205 SW, Hanbaek ST Co., Korea) operating at $100 \mathrm{rpm}$ at $37^{\circ} \mathrm{C}$. Then, $0.5 \mathrm{~mL}$ aliquots of the release media were sampled at predetermined time intervals. The amount of $\mathrm{D}$ was determined via a standardized colorimetric method using UV-Vis spectrophotometry (U-2800, PerkinElmer, USA). The D release patterns after NIR irradiation $\left(4 \mathrm{~W} \mathrm{~cm}^{-2}\right.$ for $5 \mathrm{~min}$ ) were also investigated.

\section{Bioassay \\ In vitro cytotoxicity}

The in vitro cytotoxicity of BP, BP-LH, D, and BP-DLH was assessed in MCF-7 and MDA-MB-231 (human breast adenocarcinoma) cells by a standard 3-(4,5-dimethylthiazol2-yl)-2,5-diphenyltetrazolium bromide (MTT) assay. Briefly, $2 \times 10^{4}$ viable cells per well were seeded in 96-well plates (Becton Dickinson Labware, USA), which were then incubated overnight at $37^{\circ} \mathrm{C}$. The cells were exposed to samples with chosen concentrations of equivalent $\mathrm{D}$ (or D-free samples) for $48 \mathrm{~h}$. The cells were washed, and $100 \mu \mathrm{L}$ MTT solution $\left[1.25 \mathrm{mg} \mathrm{mL}^{-1}\right.$ in Dulbecco's modified Eagle's medium (DMEM)] was added to each well. After $3 \mathrm{~h}$ incubation in the dark, the cells were lysed, and formazan crystals were dissolved using $100 \mu \mathrm{L}$ dimethyl sulfoxide. The absorbance was measured at $570 \mathrm{~nm}$ using a microplate reader (Multiskan EX, Thermo Scientific, USA). The cell viability was calculated using the following formula: cell viability $=A_{\text {sample }} / A_{\text {control }} \times 100 \%$, where $A$ is the absorbance at $570 \mathrm{~nm}$. The MTT assay of D-free samples (BP and BP-LH) was also performed after NIR irradiation $(\lambda=808$ $\mathrm{nm}$, power density $=0.8 \mathrm{~W} \mathrm{~cm}^{-2}$, exposure time $=150 \mathrm{~s}$ ).

\section{Intracellular uptake}

Cellular uptake of the samples by breast cancer cells was observed by confocal laser scanning microscopy (Leica
TCS SP2, Germany). MCF-7 and MDA-MB-231 cells (5 $\times$ $10^{4}$ cells $\mathrm{mL}^{-1}$ ) were seeded on round coverslips placed in 12-well plates and incubated for $24 \mathrm{~h}$. Coumarin-6-loaded BP-LH was added to each well. After $1 \mathrm{~h}, 100 \mathrm{ng}$ Lysotracker Red was added, and the plates were incubated for 10 min. After gently washing with PBS, the coverslips were fixed with $4 \%$ paraformaldehyde solution in the dark, mounted on glass slides, and sealed with glycerin.

The quantitative cellular uptake of the samples into the cells was determined using flow cytometry. MCF-7 and MDA-MB-231 cells $\left(2 \times 10^{5}\right)$ were seeded in 6-well plates, which were then incubated overnight. Different concentrations of BP-DLH were incubated with the cells for varying incubation times. The cells were washed twice with cold PBS, detached by trypsinization, and redispersed in $1 \mathrm{~mL}$ of cold PBS for FACS analysis (BD FACS Verse, BD Biosciences, USA). At least 10,000 events were acquired and analyzed per sample. The auto-fluorescence of untreated cells was used as an internal control.

\section{Apoptosis}

Apoptosis assays of MCF-7 and MDA-MB-231 cells were performed using a $\mathrm{PE}$-annexin $\mathrm{V} / 7$-amino actinomycin D (7-AAD) apoptosis kit (BD Biosciences, USA). Then, $3 \times 10^{5}$ cells per well were plated in a 6-well plate, which was then incubated for $12 \mathrm{~h}$. Free D and BP-DLH were then added. After $12 \mathrm{~h}$, the cells were washed with cold PBS, detached by scrapper, and redispersed in $90 \mu \mathrm{L}$ of $1 \mathrm{x}$ annexin $\mathrm{V}$ binding buffer. After this, $5 \mathrm{~mL}$ of PEannexin- $\mathrm{V}$ and 7-AAD was added, and the plate was incubated at room temperature in the dark for $10 \mathrm{~min}$. The cells were then diluted with $1 \mathrm{x}$ annexin $\mathrm{V}$ binding buffer to a final volume of $1 \mathrm{~mL}$ and analyzed using a flow cytometer (BD FACS Verse, BD Bioscience, USA). At least 10,000 events were analyzed per sample.

\section{Live/dead assay}

MCF-7 and MDA-MB-231 cells $\left(3 \times 10^{5}\right.$ cells $)$ were seeded in a 6-well plate and incubated overnight to allow cell attachment. Then, $100 \mu \mathrm{g}$ of BP-LH dispersed in PBS was added, and the plate was incubated for $3 \mathrm{~h}$ at $37^{\circ} \mathrm{C}$. The plate was washed twice with PBS, then placed under NIR laser focus and irradiated $\left(\lambda=808 \mathrm{~nm}, 4 \mathrm{~W} \mathrm{~cm}^{-2}, 3\right.$ min duration, $2 \mathrm{~mm}$ beam spot). The treated cells were washed, replaced with fresh DMEM media, and incubated for $3 \mathrm{~h}$. The cells were stained with calcein-AM (live cells, green fluorescence) and EthD-1 (dead cells, red fluorescence), and observed using inverted fluorescence microscopy (Eclipse Ti, Nikon, Japan).

\section{Cell cycle}

Changes in the cell cycle phase of MCF-7 and MDAMB-231 cells after treatment with equivalent concentrations $\left(0.1 \mu \mathrm{g} \mathrm{mL}^{-1}\right)$ of D and BP-DLH were analyzed using 
a cell-clock assay (Biocolor Ltd., UK). The cells were seeded in a six-well plate, incubated overnight at $37^{\circ} \mathrm{C}$, and treated with D and BP-DLH for $24 \mathrm{~h}$. Next, $150 \mu \mathrm{L}$ of cell-clock dye reagent was added, and the plate was incubated at $37^{\circ} \mathrm{C}$ for $1 \mathrm{~h}$ and then washed gently with PBS, after which images were taken under an optical microscope (Eclipse Ti, Nikon, Japan). The cells in G2/M, S, and G0/G1 phases were observed as stained dark blue, green, and yellow, respectively. Color pixels from at least three photomicrographs for each sample were quantified and averaged using ImageJ software.

\section{Hemolysis}

Fresh whole-blood samples from SD rats were collected in heparinized tubes and centrifuged $(3000 \times g, 10 \mathrm{~min})$, and sedimented red blood cells were resuspended in normal saline. Different concentrations of BP and BP-LH were added, and the samples were incubated at $37^{\circ} \mathrm{C}$ for $1 \mathrm{~h}$ followed by centrifugation $(3000 \times g, 10 \mathrm{~min})$. Absorbance of the supernatant was measured at $540 \mathrm{~nm}$ using a $\mathrm{UV}-\mathrm{Vis}$ spectrometer, and the percentage hemolysis was calculated as follows: percentage hemolysis $=\left(A_{\mathrm{T}}-A_{\mathrm{N}}\right) \times$ $100 /\left(A_{\mathrm{P}}-A_{\mathrm{N}}\right)$, where $A_{\mathrm{T}}, A_{\mathrm{N}}$, and $A_{\mathrm{P}}$ are the absorbances of the sample, negative control, and positive control, respectively. The negative control was untreated supernatant, while the positive control was red supernatant caused by $100 \%$ hemolysis resulting from the addition of $1 \%$ Triton X-100.

\section{Western blot}

Western blot analyses were performed to investigate the expression of different apoptotic markers. MCF-7 and MDA-MB-231 cells were seeded at a density of $4 \times 10^{5}$ and treated with their respective formulations. The cells were then lysed in ice-cold RIPA buffer (Thermo Fisher Scientific, USA), and proteins were quantified using a BSA protein assay kit. The proteins were separated by sodium dodecyl sulfate- $10 \%$ polyacrylamide gel, and were electrophoretically blotted onto a polyvinylidene fluoride membrane (Millipore, Billerica, USA). The membranes were blocked with $5 \%$ skim milk in tris-buffered saline containing $1 \%$ Tween 20, and were incubated overnight at $4{ }^{\circ} \mathrm{C}$ with primary antibodies against the proteins (Santa Cruz Biotechnology, Inc., USA). The membranes were then incubated for $1 \mathrm{~h}$ with the respective secondary antibodies and detected using chemiluminescence agents on a luminescent image analyzer (LAS-4000 mini, Fujifilm, Japan).

\section{In vivo photothermal imaging}

To measure the in vivo photothermal hyperthermia potential, MDA-MB-231-bearing xenograft mice were administered blank BP-LH dispersed in PBS via the tail vein. After $24 \mathrm{~h}$, the tumors were exposed to NIR irradiation $\left(4.0 \mathrm{~W} \mathrm{~cm}^{-2}\right)$ for up to $5 \mathrm{~min}$, and temperature contour images were recorded using a digital thermal camera (Therm-App TH, Israel) and compared with those from mice administered only saline.

\section{Antitumor activity}

MDA-MB-231 xenograft tumors were generated by the subcutaneous injection of $1 \times 10^{7}$ MDA-MB-231 cells mixed with Corning Matrigel Matrix (1:1 ratio) in the rear flanks of 5-6-week-old female BALB/c nude mice. After 2 weeks of implantation, the mice were randomly divided into 5 groups of 4-6 mice. One group was designated the control (group 1). Five groups were intravenously administered D (group 2), blank BP-LH (groups 3 and 4), and BP-DLH (groups 5 and 6) in a dose equivalent to $5 \mathrm{mg} \mathrm{D}$ $\mathrm{kg}^{-1}$ mouse body weight on days 1,4 , and 7 . The mice in groups 4 and 6 also received $808 \mathrm{~nm}$ laser irradiation (3 $\mathrm{W} \mathrm{cm}{ }^{-2}$ for $3 \mathrm{~min}$ ) through the skin on days 2,5 , and 7 . The tumor size was measured with a digital caliper, and the tumor volume was calculated as follows: volume $=1$ / $2 \times$ (longest dimension $) \times($ shortest dimension $)$. The mice were handled following the protocols approved by the Institutional Animal Ethical Committee, Yeungnam University, South Korea.

\section{Ex vivo distribution}

The in vivo distribution of $\mathrm{BP}$ and $\mathrm{BP}-\mathrm{LH}$ in the major organs of MDA-MB-231 xenograft-bearing BALB/c mice was investigated using a fluorescence in vivo imaging system (FOBI ${ }^{\mathrm{TM}}$, Neoscience, Korea). Cy5.5-conjugated BP-LH was administered intravenously via the tail vein of the mice, which were scanned at 6,12 , and $24 \mathrm{~h}$ postinjection using the red channel. The mice were then sacrificed by $\mathrm{CO}_{2}$ asphyxiation, and the tumor and major organs were excised, washed with PBS, and scanned with $\mathrm{FOBI}^{\mathrm{TM}}$. The fluorescent intensities of the tumor and organs were determined using NEOimage software.

\section{Histopathological and immunohistochemical assays}

The representative tumors from each group from the in vivo antitumor studies were excised from euthanized mice, fixed in $10 \%$ buffered formalin, sliced to $3-4 \mu \mathrm{m}$ microsections, stained with hematoxylin and eosin (H\&E), and examined under an optical microscope (Nikon, Japan) to determine the tumor cell volumes and intact tumor cell-occupied regions ( $\% \mathrm{~mm}^{-2}$ of tumor mass) using an automated image analyzer (iSolution FL ver 9.1, IMT isolution Inc., Canada). The apoptotic markers [caspase-3, cleaved poly(ADP-ribose) polymerase (PARP)], angiogenetic marker (CD31), and proliferation marker (Ki67) were determined via immunohistochemical staining using primary antisera, biotinylated secondary antibodies with avidin-biotin-peroxidase complex, and a peroxidase substrate kit (Vector Labs, USA). The mean percentage 
per $\mathrm{mm}^{2}$ region occupied by caspase-3- and PARPpositive cells and CD31- and Ki67-immunolabeled cells were measured using an automated image analyzer.

\section{Statistical analysis}

The results are reported as the mean \pm standard deviation. Student's $t$-test and one-way analysis of variance were used to determine the level of statistical significance between the groups, and the data were considered statistically significant at $p<0.05$.

\section{Results and discussion}

The BP preparations were first validated by TEM (Fig. 2a). Low-magnification TEM images revealed crumpled NSs with average lateral dimensions of $\sim 400$ $\mathrm{nm}$. High-magnification images show localized lattice structures with $d$-spacings of $0.34 \mathrm{~nm}$ and $0.22 \mathrm{~nm}$, respectively, corresponding to the (021) and (002) planes of BP, indicating that the BP NSs retained a puckered hexagonal structure. These images also revealed darker spots $(1-5 \mathrm{~nm}, N=200)$ on a BP NS, associated with the lattice fringes of $\mathrm{Au}(d$-spacing $=0.23 \mathrm{~nm})$ and $\mathrm{Sn}(d$ spacing $=0.29 \mathrm{~nm}$ ), which can be ascribed to the (111) plane of face-centered-cubic Au and the (200) plane of tetragonal Sn, respectively ${ }^{22,23}$. The XPS spectra (Fig. 2b) of the synthesized BP NSs support the co-existence of BP $(2 p)$ and $\mathrm{Au}(4 f)-\mathrm{Sn}(3 d)$, and the apparent color of the synthesized NSs was black. This implies that $\mathrm{Au}-\mathrm{Sn}$ successfully induced the conversion of RP into BP, and did not significantly perturb the original BP structure ${ }^{24}$. These results were consistent with $3 \mathrm{D}$ and 2D AFM images on mica substrates (Fig. 2c). The topological profile (in a $\mathrm{Au}-\mathrm{Sn}$ dot-free region) revealed a platelet thickness of $0.9-2.0 \mathrm{~nm}(N=5)$ on the substrate, which matched the thickness of monolayered phosphorene $(\sim 0.9 \mathrm{~nm})^{3}$. These results support the conclusion that the BP preparation consisted of single- or double-layered BP sheets; thus, the developed process was suitable for the preparation of BP NSs without the need for an exfoliation process. The AFM images also revealed the presence of bright-contrast dots on a platelet, which may have been due to $\mathrm{Au}-\mathrm{Sn}$ from the all-in-one precursor. Catalytic conversion was also demonstrated via UV-Vis (Figure S2A) and Raman (Figure S2B) spectroscopy of unreacted $(\mathrm{RP}-\mathrm{I}+\mathrm{Au}-\mathrm{Sn})$ and reacted $(\mathrm{BP})$ precursors. Although the UV-Vis spectra of both RP-I+Au-Sn and $\mathrm{BP}$ exhibited featureless absorptions, the absorption of $\mathrm{BP}$ was significantly more intense in the $300-700 \mathrm{~nm}$ region, which is consistent with the UV-to-NIR absorption characteristics of BP. The color difference (inset digital images) between the RP-I $+\mathrm{Au}-\mathrm{Sn}$ and $\mathrm{BP}$ dispersions further confirmed the catalytic conversion to produce BP. The difference between the two samples was also confirmed by Raman spectroscopy. The bands at $386 \mathrm{~cm}^{-1}$
$\left(\mathrm{A}_{\mathrm{g}}^{1}\right)$ and $471 \mathrm{~cm}^{-1}\left(\mathrm{~A}_{\mathrm{g}}^{2}\right)$ intensified after the precursor was reacted, and could be matched to the out-of-plane $\left(\sim 360 \mathrm{~cm}^{-1}\right)$ and in-plane $\left(\sim 470 \mathrm{~cm}^{-1}\right)$ modes of BP. Evidentially, $\mathrm{Au}-\mathrm{Sn}$ detached via sonication and centrifugation reveals the modes of BP. The shifts in both bands may be due to the presence of $\mathrm{Au}-\mathrm{Sn}$ and of crumpled and double-layered $\mathrm{BP}^{25}$, which interfere with the measurements and produce the characteristic bands of RP. Nevertheless, the inset optical microscopy images show different colors (bright red dots for RP-I + Au-Sn and dark gray dots for $\mathrm{BP}$ ), evidencing the conversion in the single-pass reaction. Fig. $2 \mathrm{~d}$, e show the size distributions measured by scanning mobility particle sizing (SMPS, 3936, TSI, USA; summarized in Table S1) and by DLS, respectively. The changes in size distribution between the two samples represent the conversion of RP into BP. In addition, the difference in size between SMPS and DLS measurements might be due to crumpling of the BP NSs in the gas stream, because the mean diameter measured by DLS ( $344.6 \mathrm{~nm}$ ) was similar to that observed by TEM. In addition, the mass fraction between RP-I and $\mathrm{Au}-\mathrm{Sn}$ before the conversion was found to be 9:1, which was measured using a piezobalance particle monitor (3522, Kanomax, Japan). The production yield of BP NSs was found to be $0.108 \mathrm{~g} \mathrm{~h}^{-1}$ from a single-channel reactor, which implies that employing a bundle of the reactors may produce sufficient amounts for practical application. Because of negative surface charges on the prepared $\mathrm{BP}$ NSs (-35.4 mV; Fig. 2f), an LbL process was then conducted with a cationic component [D (reaching -26.4 $\mathrm{mV}$ ) or DL (reaching $+33.3 \mathrm{mV}$ )] to load $\mathrm{H}$ (the anionic targeting component) as the outermost layer of the BP NSs. Figure S3A shows the 3D and 2D AFM topographs (with no $\mathrm{Au}-\mathrm{Sn}$ dot regions) of the BP-LH NSs, which were thicker (1.1-3.1 nm, $N=5)$ than BP NSs, likely due to a coating of LH. The opaque layer on the BP NS shown in the TEM image in Figure S3B further supports the hypothesis that the thickness increase was due to $\mathrm{LH}$ loading. A similar tendency was also observed in the TEM image of BP-DLH (Figure S3C), owing to an increase in electron density via $\mathrm{D}$ adsorption and subsequent $\mathrm{LH}$ loading via electrostatic attraction. In particular, D loading may also be promoted by weak coupling between the $p_{\mathrm{z}}$-orbitals of $\mathrm{BP}$ and the $\pi$-orbitals of $\mathrm{D}^{26}$. The $\mathrm{D}$ loading capacity was found to be $23.3 \pm 4.2 \%$, which was sufficient to be clinically acceptable. Furthermore, this was higher than that found with graphene, owing to the puckered structure of BP NSs. As shown in Fig. 2f, BP-DLH NSs possessed negative surface charges $(-29.4 \pm 1.4 \mathrm{mV})$, implying that the NSs may be suitable for CD44 receptor targeting. The outermost hydrophilic $\mathrm{H}$ layers also prevented the aggregation and subsequent precipitation of untreated BP (Figure S4), which may be due to repulsive forces between the negative charges on the NSs. Figure S4 


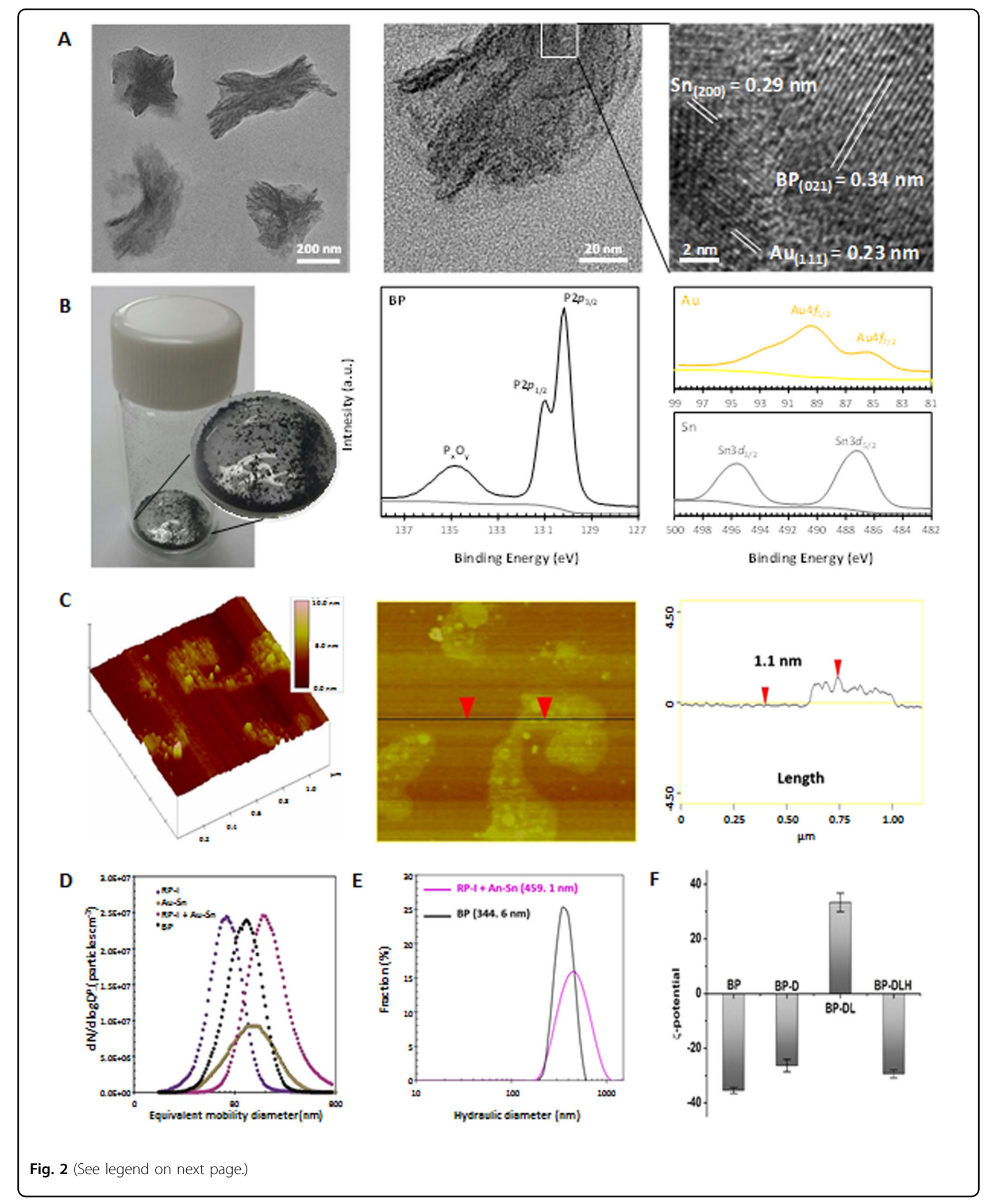


(see figure on previous page)

Fig. 2 Characterization of the single-pass processed materials. a Low- and high-magnification TEM images of the processed material. The lowmagnification image shows crumpled structures, and the high-magnification images show characteristic lattices, demonstrating the formation of BP NSs. The images also reveal Au and Sn crystallites dotted on the BP surfaces. $\mathbf{b}$ Digital images of synthesized BP NSs and detected XPS spectra of P $2 p$, $\mathrm{Au} 4 \mathrm{f}$, and Sn $3 d$ in the NSs. The XPS results also exhibit BP and Au-Sn corresponding to the high-resolution TEM image (A). $\mathbf{c} 3 \mathrm{D}$ and 2D AFM topographs with topological profiles. The dots on the planar nanostructure correspond to a Au-Sn-decorated BP NS, which was verified by TEM measurements. Size distributions of the processed material in the absence (RP-I+Au-Sn) and presence (BP) of the high-temperature (650 ${ }^{\circ} \mathrm{C}$ wall temperature) reaction measured using SMPS (d) and DLS systems (e). The SMPS measurements also show individual RP-I and Au-Sn and the hightemperature reaction-induced size redistribution. $\mathbf{f}$ Changes in the zeta potential during the LbL process. The negative surface charges of the BP NSs were suitable to electrostatically bind to amine groups of $\mathrm{D}$. $\mathrm{L}$ addition changed the surface potential to have positive polarity, which enabled subsequent binding to $\mathrm{H}$, resulting in the formation of BP-DLH

also shows the degradation of BP-LH and BP in PBS at 37 ${ }^{\circ} \mathrm{C}$, which was examined via UV-Vis and TEM measurements. The degradation rates of BP-LH and BP were 0.28 and $3.70 \mathrm{wt} \% \mathrm{~d}^{-1}$, respectively. The faster degradation of $\mathrm{BP}$ is probably due to its interaction with molecular oxygen and water to form nontoxic phosphates and phosphonates ions ${ }^{27}$. These ions are found endogenously in the body and are considered biocompatible. The $\mathrm{Au}-\mathrm{Sn}$ NPs are small enough for clearance by the renal system via glomerular filtration ${ }^{28}$.

The number of $\mathrm{L}$ and $\mathrm{H}$ layers on the BP NSs was estimated by TGA under nitrogen flow (Figure S5A). The thermograph shows that thermal decomposition in singlepass-produced BP began at $\sim 380^{\circ} \mathrm{C}$ and was near complete at $\sim 800^{\circ} \mathrm{C}$, which is consistent with the reported degradation temperature range of $500-550{ }^{\circ} \mathrm{C}^{29}$. From these analyses, the estimated $\mathrm{Au}-\mathrm{Sn}$ content of the NSs was $\sim 60 \%$, which matched the value $(58.4 \%)$ determined using a piezobalance-type aerosol particle mass monitoring system (3522, Kanomax, Japan). The TGA results of BP-L and BP-LH and their comparison to untreated BP determined the loaded $\mathrm{L}$ and $\mathrm{H}$ content to be $\sim 6 \%$ and $\sim 22 \%$, respectively.

The $\mathrm{D}$ release behavior was investigated at two different $\mathrm{pH}$ values (5.5 in ABS and 7.4 in PBS), and cumulative release after $24 \mathrm{~h}$ reached $\sim 77 \%$ and $\sim 59 \%$ at $\mathrm{pH} 5.5$ and $\mathrm{pH} 7.4$, respectively (Figure S5B), suggesting that a siteselective release of $\mathrm{D}$ (intra-tumoral hypoxic and intralysosomal/endosomal $\mathrm{pH}$ vs physiological $\mathrm{pH}$ ) can be achieved by employing BP-DLH NSs. These observations may be due to the $\mathrm{pH}$-responsive aqueous solubility of $\mathrm{D}$, including differential structural changes in the LH layers through $\mathrm{pH}$ alteration ${ }^{30,31}$. NIR $\left(\lambda=808 \mathrm{~nm}, 4 \mathrm{~W} \mathrm{~cm}^{-2}\right)$ induced stimulus for on-demand control of D release was also achieved, leading to an increase in the cumulative D release from $29 \%$ to $72 \%$ after 5 min of irradiation (Figure S5C). The photothermal activity of BP-LH NSs dispersed in Roswell Park Memorial Institute 1640 (simulated physiological medium) was also analyzed, and the dispersion temperature was monitored as a function of both BP-LH concentration (Figure S5D) and irradiation intensity (Figure S5E). Based on these analyses, both parameters proved to be workable for on-demand phototherapy via modulation of the temperature elevation upon NIR irradiation, which has been reported previously for Au nanostructures ${ }^{32}$. The surface chemistry of BP-LH was investigated by FTIR by comparing its absorption spectrum with the individual spectra of $\mathrm{BP}, \mathrm{H}$, and $\mathrm{L}$ (Figure S5F). BP showed a broad band at $\sim 1050 \mathrm{~cm}^{-1}$ and a small band centered at $\sim 1630 \mathrm{~cm}^{-1}$, which could be assigned to the $\mathrm{P}-\mathrm{O}$ and $\mathrm{P}=\mathrm{O}$ stretching modes, respectively ${ }^{7} . \mathrm{H}$ showed intra- and inter-molecular $-\mathrm{OH}$ stretching vibrations from alcohols and hydrogen-bonded -NH groups, represented by the broad peak centered at $\sim 3340 \mathrm{~cm}^{-1}$. The band at $1050 \mathrm{~cm}^{-1}$ is related to the hemiacetalic $\mathrm{C}-\mathrm{O}-\mathrm{C}$ of saccharides, while the bands at $1620 \mathrm{~cm}^{-1}$ and $1520 \mathrm{~cm}^{-1}$ can be assigned to the symmetric and asymmetric vibrations of COO- groups, respectively $^{33}$. L exhibited characteristic bands of $-\mathrm{NH}_{2}(\mathrm{C}=\mathrm{O})$ symmetric vibrational I and II amides at $\sim 1650 \mathrm{~cm}^{-1}$ and $\sim 1540 \mathrm{~cm}^{-1}$, respectively ${ }^{34}$. The peak at $2910 \mathrm{~cm}^{-1}$ could be ascribed to alkyl vibrations. BP-LH showed the combined spectrum of $\mathrm{L}$ and $\mathrm{H}$. In particular, the signal intensity for saccharides was the strongest, while the bands characteristic of $\mathrm{L}$ were muted ${ }^{35}$, showing a significantly greater amount of $\mathrm{H}$ than $\mathrm{L}$ on the surfaces of BP NSs. There was a superimposition of the $\mathrm{P}-\mathrm{O}$ band from BP with the saccharide bands of $\mathrm{H}$ and $\mathrm{L}$, which produced a large shoulder to the band at $3400 \mathrm{~cm}^{-1}$.

The photothermal activity of BP-LH in killing breast cancer cells (MCF-7 and MDA-MB-231) was determined by MTT assay, and the results were compared to those of free D (Fig. 3a, b). The viability of both cell lines remained $>80 \%$ when treated with BP-LH NSs at different concentrations, indicating that the NSs were biocompatible and warranting further investigation into their therapeutic efficacy. NIR irradiation $\left(\lambda=808 \mathrm{~nm}, \mathrm{~W} \mathrm{~cm}^{-2}, 3 \mathrm{~min}\right)$ of BP-LH significantly enhanced its cytotoxic effect even in the absence of $\mathrm{D}$, which implies that the BP preparation can be employed as a phototransducer upon incubation with cancer cells (insets of Fig. 3a, b). The D-containing formulation (i.e., BP-DLH) exhibited comparable dose- 


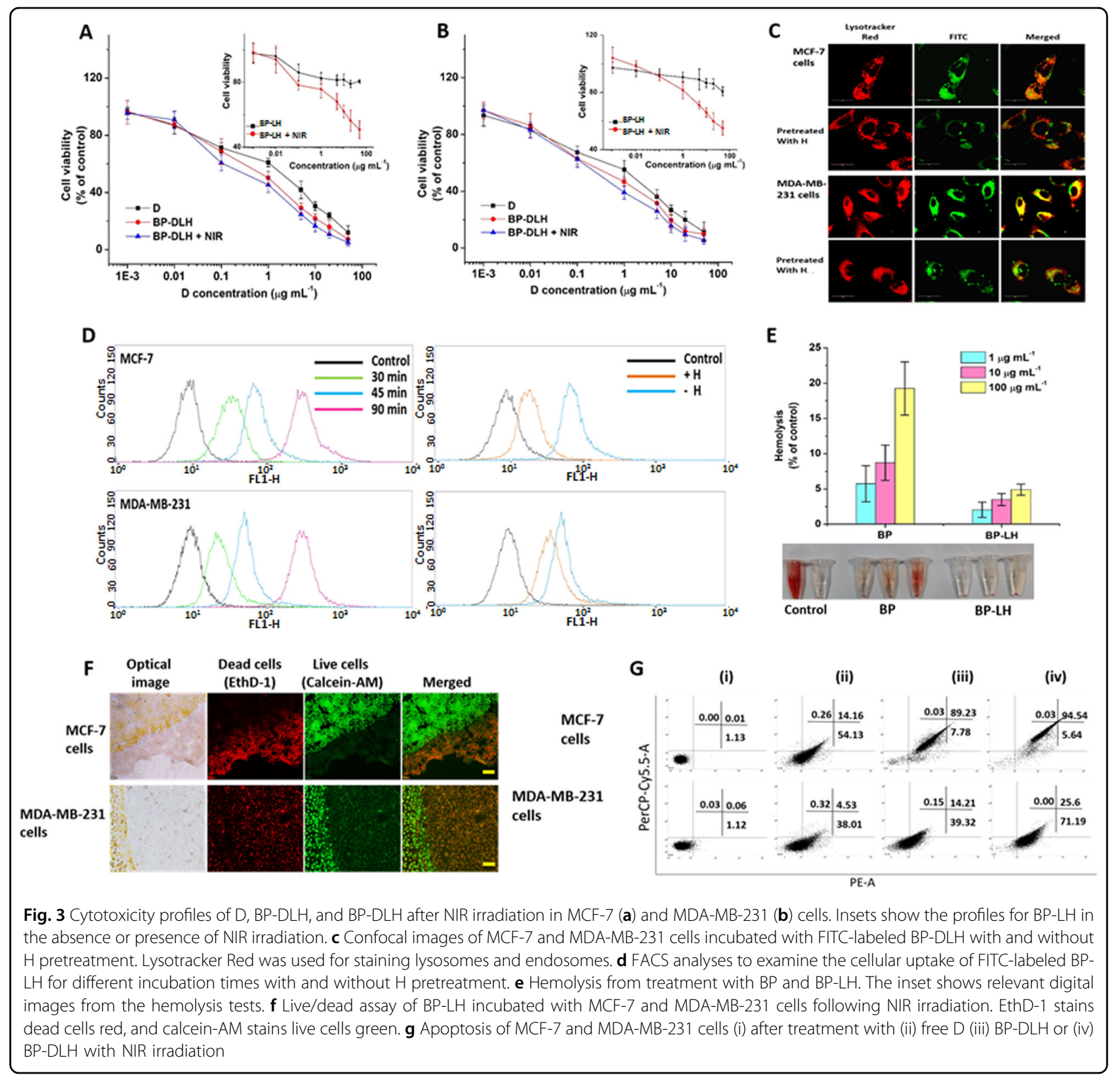

dependent cytotoxicity to free D in both cell lines. The cytotoxic effect of BP-DLH was further enhanced in both cell lines upon NIR irradiation ( $>95 \%$ cytotoxicity at 50 $\left.\mu \mathrm{g} \mathrm{mL}^{-1}\right)$. In particular, the increase in cytotoxicity compared to free $\mathrm{D}$ was substantial when the $\mathrm{D}$ concentration was $>0.1 \mu \mathrm{g} \mathrm{mL}^{-1}$, probably due to NIR-induced enhanced D uptake and sensitivity from alterations of the cellular membrane permeability ${ }^{36}$. The cancer targeting activity and cellular internalization via CD44 receptor-mediated endocytosis were investigated by confocal microscopy and flow cytometry. Fluorescein isothiocyanate (FITC)-conjugated BP-LH exhibited primary internalization into acidic compartments, as evidenced by the co-localization of the green fluorescent signal of BP-LH with the red fluorescence of Lysotracker Red (a weakly basic amine dye that stains endosomal and lysosomal vesicles; Fig. 3c). To verify the CD44 receptor-specific internalization of BP$\mathrm{LH}$, a competitive binding assay was conducted by pretreating the cells with excess $\mathrm{H}$ to saturate the receptors prior to BP-LH incubation. This pretreatment significantly diminished the green fluorescence intensity of FITC-conjugated BP-LH, indicating that intracellular internalization occurs primarily via $\mathrm{CD} 44$ receptormediated endocytosis because excess $\mathrm{H}$ competes with $\mathrm{BP}-\mathrm{LH}$ for receptor binding sites. These findings were quantitatively supported by flow cytometry, which showed 
that cellular uptake was a function of incubation time (Fig. 3d). Compared to normal cells, $\mathrm{H}$ pretreated cells showed smaller rightward shifts in fluorescent signals, even though the concentration and incubation time were kept constant. These data reflect the preferential targetability and intracellular accumulation of H-loaded BP NSs via CD44 receptor-mediated endocytosis. In addition, MCF-7 cells expressed relatively lower levels of CD44 receptors than MDA-MB-231 cells, which is in agreement with a previous report ${ }^{37}$.

A hemolysis assay was performed to further validate the biological safety of BP NSs (Fig. 3e). Compared to BP alone, BP-LH NSs did not induce significant hemolysis $(<5 \%)$ when NSs were co-cultured with red blood cells in a concentration range of $1-100 \mu \mathrm{g} \mathrm{mL}^{-1}$. This implies that LH loading is hemoprotective and can enhance the biocompatibility of the BP NSs produced in this study, increasing the possibility for their use as cancer therapeutics.

The in vitro photothermal efficacy of BP-LH NSs upon NIR irradiation with MCF-7 and MDA-MB-231 cells was further investigated using an EthD-1/Calcein-AM live/ dead assay (Fig. 3f). Live cells were stained green (with Calcein-AM), whereas dead cells were stained red (with EthD-1) and were localized to the circular laser spot $(\sim 2$ $\mathrm{mm}$ diameter), implying that NIR irradiation can thermally ablate cancer cells, even in the absence of D. The clearly delineated boundary of the dead cell region suggests minimal risk of off-target hyperthermia-induced cell necrosis of surrounding healthy tissues. A flow cytometric PE-annexin V/7-AAD apoptosis assay was performed to quantify and compare the apoptotic subpopulation of cells after incubation with free D and BP-DLH (Fig. 3g). In MCF-7 cells, free D induced early apoptosis in $54 \%$ of cells, while BP-DLH containing an equivalent amount of D shifted a larger fraction (89\%) to late apoptosis. Although free D-treated MDA-MB-231 cells contained a larger fraction in early apoptosis (38\%), BP-DLH preferentially increased the early apoptotic fraction over the late fraction. When BP-DLH-treated cells were irradiated by an NIR laser, the apoptotic fraction of cells increased synergistically, while the necrotic fraction remained negligible. Apoptotic modes for cancer cell death are preferred because necrosis can trigger detrimental inflammatory and immunogenic responses, while apoptotic cells are mostly cleared by phagocytosis in vivo ${ }^{38}$.

The apoptosis of cancer cells by BP-DLH and its augmentation by NIR irradiation were further confirmed by western blot analyses (Figure S6A). Compared to free D, BP-DLH treatment increased the expression of the proapoptotic proteins $\mathrm{BAX}$ and $\mathrm{p} 53$, but reduced the expression of the anti-apoptotic marker Bcl-2 in MCF-7 cells. Similarly, BAX expression increased in MDA-MB231 cells, and Bcl-2 expression decreased. This suggests that BP-LH containing D enhanced apoptosis in cancer cells, which was further augmented by NIR irradiation. Cell cycle perturbation analyses of MCF-7 and MDA-MB231 cells after treatment with free D and BP-DLH were performed using a redox dye (Figures S6B and S6C). In MCF-7 cells, free D induced cell cycle arrest primarily at the G1 check point and partly at the G2M check point, while BP-DLH significantly enhanced the subpopulation of cells mostly at G2M and partly at G1. In MDA-MB-231 cells, both free D and BP-DLH induced G2M arrest.

MDA-MB-231 xenografted BALB/c mice were administered BP-LH intravenously (i.v.), and $24 \mathrm{~h}$ post-injection, the tumor was irradiated with an NIR laser $(2.5 \mathrm{~W}$ $\mathrm{cm}^{-2}$ ) for $5 \mathrm{~min}$ to assess the in vivo photothermal efficacy of the BP-LH NSs (Figure S7). The thermographs revealed linear temperature increments (up to $48.8^{\circ} \mathrm{C}$ after $5 \mathrm{~min}$ ) in the tumor focal region of BP-LH-administered mice, with no concomitant temperature elevation in surrounding tissues and organs, demonstrating a highly selective and minimally invasive application for superficial (up to $10 \mathrm{~mm}$ deep) solid tumors ${ }^{36}$. The control mice that were administered saline did not exhibit significant temperature elevation at the beam spot $\left(4^{\circ} \mathrm{C}\right)$.

An in vivo antitumor study in subcutaneous MDA-MB231 xenograft-bearing nude mice showed significant regression in tumor burden upon three cycles of i.v. administration of BP-DLH (with concurrent NIR irradiation $24 \mathrm{~h}$ post-injection) compared to other mice groups that were administered free D and BP-DLH (without NIR irradiation). Body weight monitoring (Fig. 4a) determined that free D induced marked weight loss starting at $5 \mathrm{~d}$ post-treatment (two out of six mice in the $\mathrm{D}$ group died at $24 \mathrm{~d}$ ), probably due to the cardiotoxic and myelosuppressive properties of $\mathrm{D}^{39}$, whereas other treatments using BP-LH and BP-DLH with and without NIR irradiation did not produce significant losses in body weight by the end of the experiment $(24 \mathrm{~d})$. The treated mice were normal and did not exhibit any impaired mobility or infection, suggesting minimal acute toxicity. The mean tumor volume was in the order of BP-DLH $+\mathrm{NIR}<\mathrm{BP}-\mathrm{DLH}<$ free $\mathrm{D}<\mathrm{BP}-\mathrm{LH}+\mathrm{NIR}<\mathrm{BP}-\mathrm{LH}<$ control (Fig. 4b). These tumor volume findings were further corroborated by measurement of the mean tumor weight of mice in different groups (Fig. 4c; $N=3$ ). Free D was critical in significantly reducing tumor weight, and the reduction was further enhanced when $\mathrm{D}$ was combined with non-systemic cytotoxic BP-LH and NIR. The logrank test (Figure S8A) did not exhibit a significant difference between the survival percentages of treated mice $(p<0.06)$, and the survival periods were consistent with the tumor volumes (Figure S8B); thus, BP-DLH+NIR induced the longest survival period. A BP-DLH biodistribution study of Cy5.5-labeled BP and BP-LH was performed in MDA-MB-231 xenografted tumor-bearing 


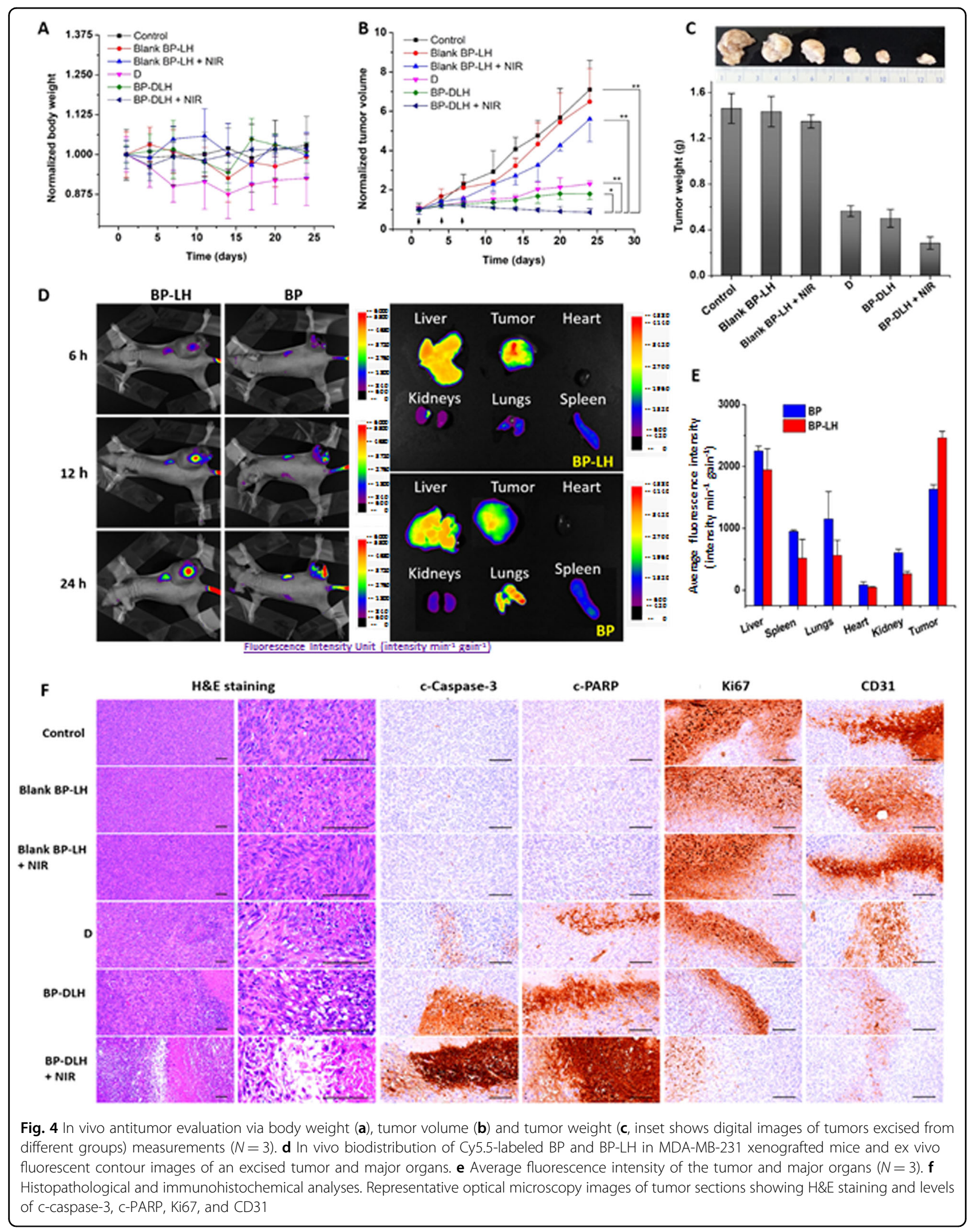


mice following i.v. administration (Fig. 4d). Real-time live imaging showed that labeled BP-LH progressively accumulated in MDA-MB-231 tumors after $24 \mathrm{~h}$. The distinguishable tumor foci with clear boundaries with surrounding subcutaneous tissue at $24 \mathrm{~h}$ suggest the tumor-targeting capability of the BP-LH NSs. The untreated BP NSs also exhibited a similar pattern of accumulation in tumors, which is attributable to passive targeting via enhanced permeability and retention effects. However, the relatively lower fluorescence intensity and the lack of delineating boundaries around tumors show that untreated NSs are also internalized by surrounding non-tumor tissues. Ex vivo imaging was performed on tumors and major organs excised from these mice. Both BP-LH and untreated BP NSs localized at the liver, lungs, kidneys, heart and spleen. BP-LH-administered mice exhibited strong fluorescence in tumors compared with those treated with BP alone (Fig. 4e). It should be emphasized that hearts showed minimal bioaccumulation, which is clinically relevant to the delivery of highly cardiotoxic drugs, such as $\mathrm{D}^{39}$. Histological changes in tumors dissected from different groups of mice from the antitumor study were analyzed using hematoxylin and eosin (H\&E) staining (Fig. 4f). Tumor sections of BPDLH- and NIR-treated mice showed drastic microstructural alterations, such as apoptotic condensation, cellular fragmentation, and necrosis, whereas untreated control samples retained their morphology, and no nuclear atypia was observed. Immunohistological analysis revealed increased apoptotic markers, such as c-caspase- 3 and c-PARP, whereas angiogenetic markers, such as CD31, and the proliferation marker (Ki67), decreased in the tumor samples of mice treated with BP-DLH and NIR compared with the control and other treatment groups. Significant $(p<0.01)$ decreases in tumor cell volume, CD31- and Ki67-positive cells and increases in c-caspase3 and c-PARP immunoreactive cells were demonstrated in all three D-based formula-treated tumor masses, in the order of $\mathrm{BP}-\mathrm{DLH}+\mathrm{NIR}>\mathrm{BP}-\mathrm{DLH}>\mathrm{D}$, compared with the untreated control masses (Table S2). These results suggest that D loading with $\mathrm{LH}$ onto BP NSs could be a suitable future platform for therapeutics against breast cancer, particularly when combined with advanced photoirradiation systems for cancer treatment.

To facilitate the clinical translation of BP NSs in a timely manner, the present study proposes a single-pass continuous route to produce BP NSs as base materials for cancer therapeutics. NSs were specifically loaded with DLH using an electrostatic LbL assembly method, avoiding the need for further processing of the NSs (i.e., purification, exfoliation or functionalization). These NSs were effective in the targeted chemo-phototherapy of CD44-overexpressing breast cancer cells. BP produced by the reported single-pass catalytic conversion possessed lateral nanodimensions, biocompatibility, photothermal activity, drug-loading availability, and targeting components and thus warrants consideration as an advanced cancer therapeutic. This study not only provides a practical and green route for the continuous production of $\mathrm{BP}$ NSs but also supports their potential as future cancer therapeutics; therefore, this work may be a stepping stone to opening new horizons for the medical use of these $2 \mathrm{D}$ materials.

\section{Acknowledgements \\ This research was supported by Basic Science Research Program through the National Research Foundation of Korea (NRF) funded by the Ministry of Science, ICT and future Planning (NRF-2018R1A2A1A05020683). This work was also supported by the NRF of Korea Grant funded by the Korean Government (NRF-2018R1A2A2A05021143). \\ Author details \\ ${ }^{1}$ School of Mechanical Engineering, Yonsei University, Seoul 03722, Republic of Korea. ${ }^{2}$ College of Korean Medicine, Daegu Haany University, Gyeongsan 38610, Republic of Korea. ${ }^{3}$ College of Pharmacy, Yeungnam University, Gyeongsan 38541, Republic of Korea. ${ }^{4}$ School of Mechanical Engineering, Yeungnam University, Gyeongsan 38541, Republic of Korea}

\section{Author contributions}

B.K.P. performed the experiments and wrote the manuscript. S.K.K. performed the experiments. J.H.B., J.O.K., and J.H. conceived of the project, designed and performed the experiments, and wrote the manuscript.

\section{Conflict of interest}

The authors declare that they have no conflict of interest.

\section{Publisher's note}

Springer Nature remains neutral with regard to jurisdictional claims in published maps and institutional affiliations.

Supplementary information is available for this paper at https://doi.org/ 10.1038/s41427-018-0068-8.

Received: 29 April 2018 Revised: 9 June 2018 Accepted: 13 June 2018. Published online: 7 August 2018

\section{References}

1. Liu, B., Abbas, A. \& Zhou, C. Two-dimensional semiconductors: from materials preparation to electronic applications. Adv. Electron. Mater. 3, 1700045 (2017).

2. Molle, A. et al. Buckled two-dimensional xene sheets. Nat. Mater. 16, 163-169 (2017).

3. Liu, H. et al. Phosphorene: an unexplored $2 \mathrm{D}$ semiconductor with a high hole mobility. ACS Nano 8, 4033-4041 (2014).

4. Li, L. et al. Black phosphorus field-effect transistors. Nat. Nanotechnol. 9, 372-377 (2014)

5. Radisavljevic, B., Radenovic, A., Brivio, J., Giacometti, V. \& Kis, A. Single-layer $\mathrm{MoS}_{2}$ transistors. Nat. Nanotechnol. 6, 147-150 (2011).

6. Schütz, M., Maschio, L., Karttunen, A. J. \& Usvyat, D. Exfoliation energy of black phosphorus revisited: a coupled cluster benchmark. J. Phys. Chem. Lett. 8 1290-1294 (2017).

7. Sun, C. X. et al. One-pot solventless preparation of PEGylated black phosphorus nanoparticles for photoacoustic imaging and photothermal therapy of cancer. Biomaterials 91, 81-89 (2016).

8. Yang, G. et al. Facile synthesis of black phosphorus-Au nanocomposites for enhanced photothermal cancer therapy and surface-enhanced Raman scattering analysis. Biomater. Sci. 5, 2048-2055 (2017).

9. Wang, H. et al. Ultrathin black phosphorus nanosheets for efficient singlet oxygen generation. J. Am. Chem. Soc. 137, 11376-11382 (2015). 
10. Chen, W. et al. Black phosphorus nanosheet-based drug delivery system for synergistic photodynamic/photothermal/chemotherapy of cancer. Adv. Mater. 29, 1603864 (2017).

11. Fojtů, M., Chia, X., Sofer, Z., Masařík, M. \& Pumera, M. Black phosphorus nanoparticles potentiate the anticancer effect of oxaliplatin in ovarian cancer cell line. Adv. Funct. Mater. 27, 1701955 (2017).

12. Nilges, T., Kersting, M. \& Pfeifer, T. A fast low-pressure transport route to large black phosphorus single crystals. J. Solid State Chem. 181, 1707-1711 (2008).

13. Zhao, M. et al. Growth mechanism and enhanced yield of black phosphorus microribbons. Cryst. Growth Des. 16, 1096-1103 (2016).

14. Zhang, Y. et al. Exfoliation of stable 2D black phosphorus for device fabrication. Chem. Mater. 29, 6445-6456 (2017).

15. Woomer, A. H. et al. Phosphorene: synthesis, scale-up, and quantitative optical spectroscopy. ACS Nano 9, 8869-8884 (2015).

16. Boukhvalov, D., Rudenko, A., Prishchenko, D., Mazurenko, V. \& Katsnelson, M. Chemical modifications and stability of phosphorene with impurities: a first principles study. Phys. Chem. Chem. Phys. 17, 15209-15217 (2015).

17. Sofer, Z., Bousa, D., Luxa, J., Mazanek, V. \& Pumera, M. Few-layer black phosphorus nanoparticles. Chem. Commun. 52, 1563-1566 (2016).

18. Visvader, J. E. \& Lindeman, G. J. Cancer stem cells in solid tumours: accumulating evidence and unresolved questions. Nat. Rev. Cancer 8, 755-768 (2008)

19. Bachar, G. et al. Hyaluronan-grafted particle clusters loaded with mitomycin C as selective nanovectors for primary head and neck cancers. Biomaterials $\mathbf{3 2}$ 4840-4848 (2011).

20. Eliaz, R. E. \& Szoka, F. C. Jr. Liposome-encapsulated doxorubicin targeted to CD44: a strategy to kill CD44-overexpressing tumor cells. Cancer Res. 61 2592-2601 (2001)

21. Schäfer, $H$. In chemische transportreaktionen in monographien zu angewandte chemie und chemie-ingenieur-technik. Vol. 76, p. 67, p. 885 (VCH, Weinheim, Germany, 1962)

22. Caballero, A., Morales, J. \& Sanchez, L. Tin nanoparticles formed in the presence of cellulose fibers exhibit excellent electrochemical performance as anode materials in lithium-ion batteries. Electrochem. Solid. Lett. 8, A464 (2005).

23. Byeon, J. H. \& Roberts, J. T. Photoionization of nanosized aerosol gold agglomerates and their deposition to form nanoscale islands on substrates. Langmuir 30, 8770-8775 (2014).

24. Ge, S., Zhang, L., Wang, P. \& Fang, Y. Intense, stable and excitation wavelengthindependent photoluminescence emission in the blue-violet region from phosphorene quantum Dots. Sci. Rep. 6, 27307 (2016).

25. Wang, G., Loh, G. C., Pandey, R. \& Karna, S. P. Out-of-plane structural flexibility of phosphorene. Nanotechnology 27, 055701 (2016).
26. Cai, Y., Zhang, G. \& Zhang, Y. -W. Electronic properties of phosphorene/ graphene and phosphorene/hexagonal boron nitride heterostructures. J. Phys. Chem. C. 119, 13929-13936 (2015).

27. Huang, Y. et al. Interaction of black phosphorus with oxygen and water. Chem Mater. 28, 8330-8339 (2016).

28. Liu, J. B., Yu, M. X., Zhou, C. \& Zheng, J. Renal clearable inorganic nanoparticles: a new frontier of bionanotechnology. Mater. Today 16, 477-486 (2013).

29. Passaglia, E. et al. Novel polystyrene-based nanocomposites by phosphorene dispersion. RSC Adv. 6, 53777-53783 (2016).

30. Poudel, B. K., Kim, J. O. \& Byeon, J. H. Photoinduced rapid transformation from Au nanoagglomerates to drug-conjugated Au nanovesicles. Adv. Sci. 5 , 1700563 (2018).

31. Dreaden, E. C. et al. Bimodal tumor-targeting from microenvironment responsive hyaluronan layer-by-layer (LbL) nanoparticles. ACS Nano 8 8374-8382 (2014)

32. Sun, Z. et al. K. Ultrasmall black phosphorus quantum dots: synthesis and use as photothermal agents. Angew. Chem. Int. Ed. 54, 11526-11530 (2015).

33. Leone, L., Consumi, M., Lamponi, S. \& Magnani, A. New hyaluronan derivative with prolonged half-life for ophthalmogical formulation. Carbohydr. Polym. 88, 799-808 (2012).

34. Rozenberg, M. \& Shoham, G. FIIR spectra of solid poly--lysine in the stretching $\mathrm{NH}$ mode range. Biophys. Chem. 125, 166-171 (2017).

35. Shen, L., Chaudouet, P., Ji, J. \& Picart, C. pH-amplified multilayer films based on hyaluronan: Influence of HA molecular weight and concentration on film growth and stability. Biomacromolecules 12, 1322-1331 (2011).

36. Poudel, B. K. et al. PEGylated thermosensitive lipid-coated hollow gold nanoshells for effective combinational chemo-photothermal therapy of pancreatic cancer. Colloids Surf. B Biointerfaces 160, 73-83 (2017).

37. Qhattal, H. S. \& Liu, X. Characterization of CD44-mediated cancer cell uptake and intracellular distribution of hyaluronan-grafted liposomes. Mol. Pharm. 8, 1233-1246 (2011)

38. Melamed, J. R., Edelstein, R. S. \& Day, E. S. Elucidating the fundamental mechanisms of cell death triggered by photothermal therapy. ACS Nano $\mathbf{9}$ 6-11 (2015).

39. Shafei, A. et al. A review on the efficacy and toxicity of different doxorubicin nanoparticles for targeted therapy in metastatic breast cancer. Biomed. Pharmacother. 95, 1209-1218 (2017). 